\title{
Special issue on the Trilinos project, Part 1 of 2
}

\author{
Michael A. Heroux * and Damian Rouson \\ Sandia National Laboratories, Albuquerque, NM, USA
}

The Trilinos project has been under development for about one decade. In that time it has grown from a small solvers project to a large, comprehensive library development effort for scalable computational science and engineering applications. In this collection of articles we present a broad discussion of many of the recent developments in Trilinos.

The special issue is divided into two journal issues, the first issue (Scientific Programming, Volume 20, Number 2, 2012) contains an updated overview of the project and a collection of papers discussing tools to set up and manage data and problem definition. With this collection of tools, users can quickly build problem definitions and set up data needed by the solvers.
The second issue (Scientific Programming, Volume 20 , Number 3,2012 ) contains papers on the next generation of equation solvers in Trilinos. These new solvers encode the latest in solver algorithms and are designed for execution on scalable manycore systems. Part two of this issue also includes a discussion of four problem solving environments that present a certain perspective on the capabilities in Trilinos, providing users with a high-level environment that caters to specific problem domains.

\footnotetext{
* Corresponding author: Michael A. Heroux, Sandia National Laboratories, P.O. Box 5800, Albuquerque, NM 87185-1110, USA. Tel.: +1 320845 7695; E-mail: maherou@ sandia.gov.
} 

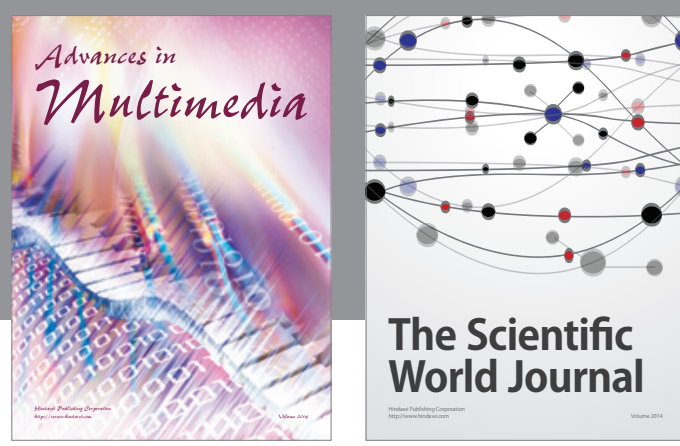

The Scientific World Journal
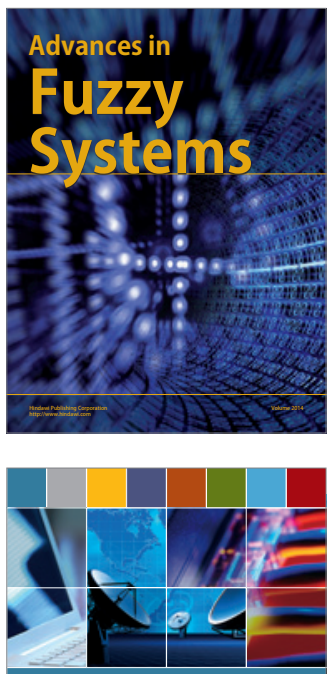

Computer Networks and Communications
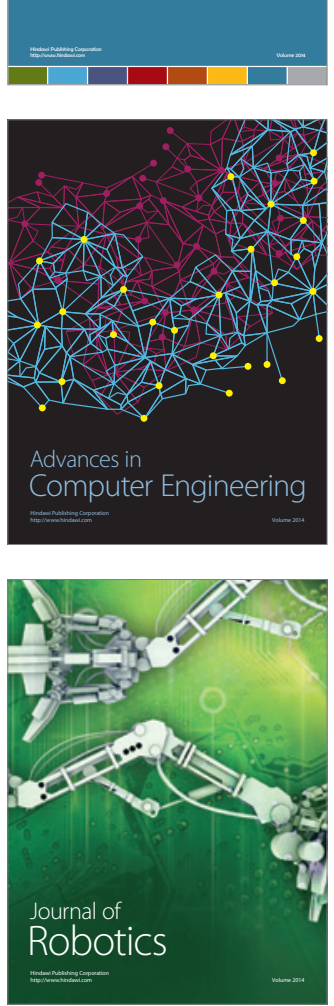
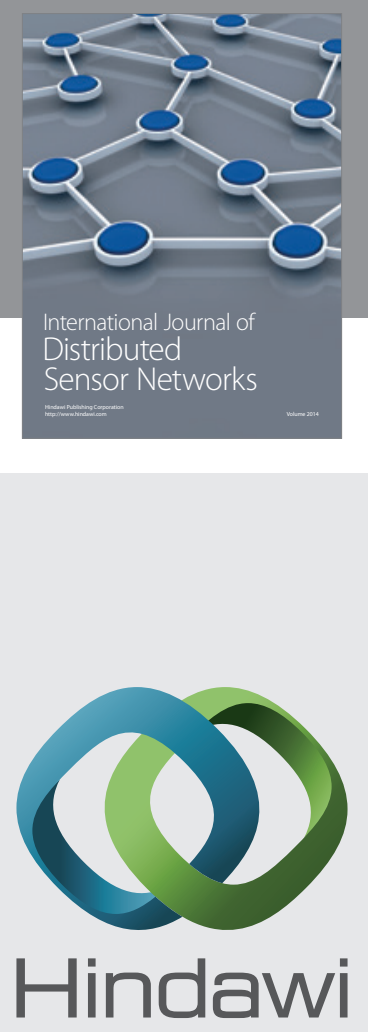

Submit your manuscripts at

http://www.hindawi.com
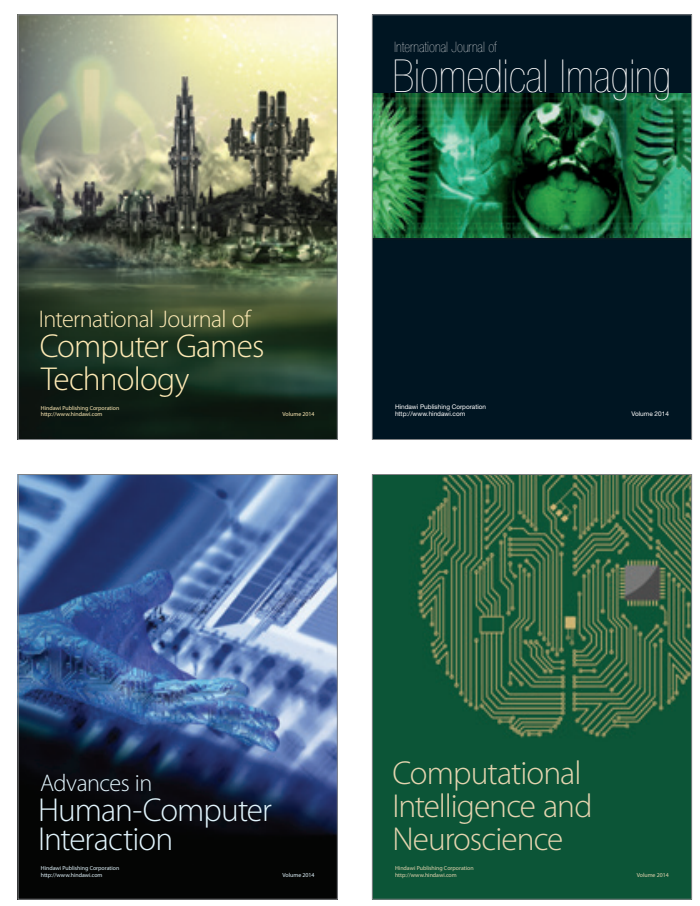
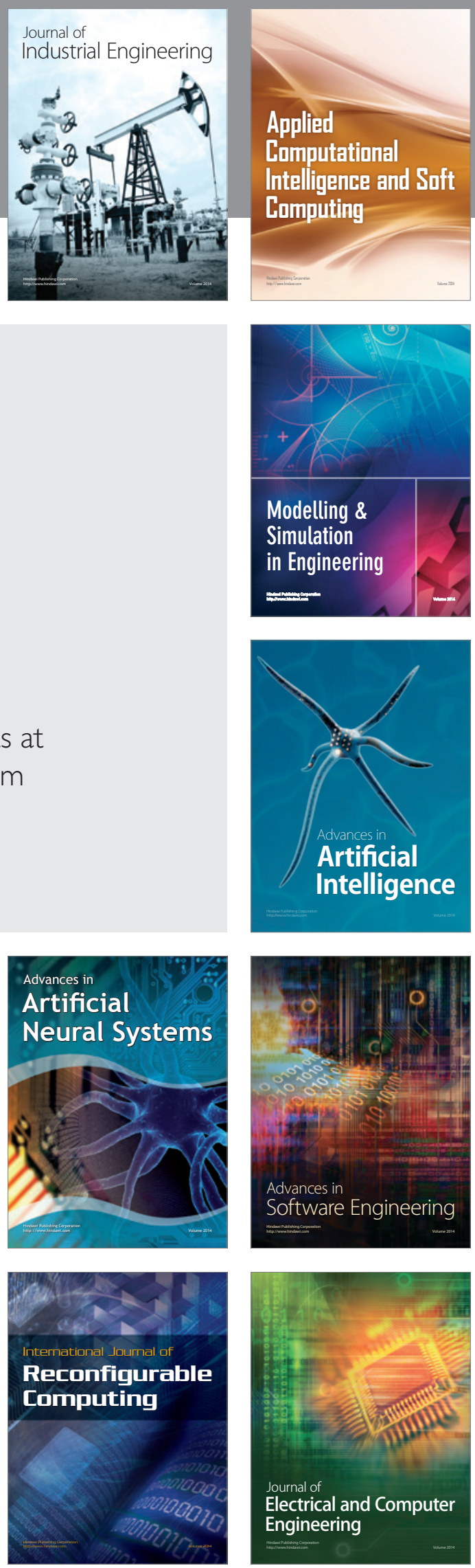